OPEN

SUBJECT AREAS:

ANIMAL BREEDING

GENETIC ENGINEERING

Received

14 August 2014

Accepted

3 December 2014

Published

5 January 2015

Correspondence and requests for materials should be addressed to W.H. (huwei@ihb.ac.

$\mathrm{cn})$

\section{A controllable on-off strategy for the reproductive containment of fish}

\author{
Yunsheng Zhang ${ }^{1,2}$, Ji Chen' ${ }^{1}$ Xiaojuan Cui ${ }^{1,2}$, Daji Luo ${ }^{1,3}$, Hui Xia ${ }^{1,2}$, Jun Dai', Zuoyan Zhu' \& Wei Hu'
}

'State Key Laboratory of Freshwater Ecology and Biotechnology, Institute of Hydrobiology, Chinese Academy of Sciences, No. 7 Donghu South Road, Wuhan 430072, China, ${ }^{2}$ University of Chinese Academy of Sciences, Beijing 100049, China, ${ }^{3}$ Department of Genetics, School of Basic Medical Sciences, Wuhan University, No. 185 Donghu East Road, Wuhan 430071, China.

A major impediment to the commercialization and cultivation of transgenic fish is the potential ecological risks they pose to natural environments: a problem that could be solved by the production of sterile transgenic fish. Here, we have developed an on-off reproductive containment strategy for fish that renders the offspring sterile but leaves their parents fertile. TG1 (Tol2-CMV-GFP-pA-CMV-gal4-pA-Tol2) and TG2 (Tol2-CMV-RFP-pA-5 $\times$ UAS-as/dnd-pA-Tol2) zebrafish lines were established using a GAL4/UAS system. While the parental lines remained fertile, in the hybrid offspring, GAL4 induced $5 \times$ UAS to drive the transcription of antisense $d n d$, which significantly down-regulated endogenous $d n d$ expression. This disrupted the migration of primordial germ cells (PGCs), led to their apoptosis, and resulted in few or no PGCs migrating to the genital ridge. This process induced sterility or reduced fertility in adult fish. This on-off strategy is a potentially effective means of generating sterile fish for commercialization while retaining fertility in brood stocks, and offers a novel method to mitigate the ecological risks of fish introductions.

F ish are the last wild food available to humans ${ }^{1}$. However, wild fish stocks are under increasing pressure from overfishing as the global demand for fish increases alongside rapid population growth. Overfishing compromises the long-term sustainability of fisheries resources and results in biodiversity loss, potentially leading to ecosystem collapse ${ }^{2,3}$. Aquaculture is, therefore, considered to be the only long-term sustainable solution to supply our growing demand for fish ${ }^{4}$. The introduction of exotic fish species to the environment is an effective method of sourcing fish with valuable traits for aquaculture, but there is an ecological risk associated with it: the introduction of the exotic species may be economically viable but can also cause substantial economic and ecological damage should the species become invasive. Transgenic technologies offer another means of producing new fish varieties that exhibit physiologically and commercially desirable traits for aquaculture, and are an important factor in the sustainable development of future aquaculture industries. Since the first rapidgrowth transgenic fish in the world was developed by our group 30 years ago ${ }^{5}$, many fish breeds with commercially desirable traits (e.g. rapid growth, cold tolerance, enhanced disease resistance) have been generated using transgenic technologies ${ }^{6,7}$. However, to date, no transgenic fish has been approved for release into a natural environment, or for commercial cultivation as food. The "AquaBounty ${ }^{\mathrm{TM}}$ " all-fish growth-hormone transgenic salmon (Oncorhynchus tshawytscha) is perhaps the most notable example of current attempts to commercialize the cultivation of transgenic fish. This variety was declared safe for consumption by the US Food and Drug Administration in 2010, and is nearing approval for commercialization. The primary impediment to the commercialization of transgenic fish is the concern over their potential ecological risk to natural ecosystems. As a result of their superior viability and competitive ability, the inadvertent release or escape of transgenic fish into natural environments can alter natural community structure. Additionally, transgenic fish may also interbreed with native fish populations resulting in gene introgression to the wild.

A key means of eliminating the potential ecological risk posed by invasive and transgenic fish is fertility control to make individuals infertile ${ }^{9-15}$. At present, however, there are few cost-effective means of controlling reproduction in fish. Generating fish that are triploid rather than diploid is currently the most common strategy used to develop sterile fish. However, in commercially important species, the 'triploidization' rate (achieved via physical or chemical methods) rarely approaches $100 \%$, and varies greatly among species, treatment methods and egg quality $^{16}$. Although up to $99.8 \%$ of the all-female transgenic salmon produced by AquaBounty are triploid and sterile, some researchers are still concerned about the non-zero possibility that fertile escapees will be produced ${ }^{17}$. Moreover, in some fish species triploidization is known to produce defects such as impaired growth and reduced disease resistance ${ }^{16}$. For example, compared with diploids, the growth rate of triploid tilalia (Oreochromis 
mossambicus) was slow ${ }^{18}$, and triploid rainbow trout and Atlantic salmon were more susceptible to disease ${ }^{19,20}$. The death rate of triploid Atlantic salmon was higher than that of the diploids in adverse conditions such as high temperature and hypoxia ${ }^{21}$. Therefore, developing new strategies for the reproductive containment of fish is the focus of extensive research with promising outcomes both for enabling the commercialization of transgenic fish and for protecting aquatic ecosystems.

Recently, the P0 generation of sterile transgenic common carp (Cyprinus carpio L.) and tilapia (Oreochromis sp.) were produced, both of which achieve sterility via the expression of antisense gonadotropin-releasing hormone $(G n R H)$ RNA to inhibit the expression of endogenous $G n R H^{22-24}$. Unfortunately, the sterile trait in these transgenic P0 founders is not heritable. The development of transgenic fish varieties in which sterility can be maintained as a heritable trait is critical for large-scale aquaculture. Wong and Collodi (2013) recently described a method to induce sterilization of zebrafish with heat shock treatment ${ }^{25}$. In this method the overexpression of the Stromal-derived factor 1a gene ( $s d f 1 a)$ driven by the heat-shock protein-70 promoter could impair the migration of PGCs during embryogenesis, such that the embryos developed into sterile adults ${ }^{25}$. However, heat shock treatment is not not convenient and practical for large-scale fish production in aquaculture. Hu et al., (2010) established an inducible platform to control the reproduction of transgenic ornamental zebrafish using the Escherichia coli nitroreductase (NTR)/metronidazole (Mtz) system ${ }^{26}$. It should be noted that this platform is prodrug-dependent. In their system, an NTR-mediated germ cell ablation occurred only if the transgenic fish were raised in an Mtz solution for at least two weeks ${ }^{26}$. Mtz is an aquatic pollutant and the induced cytotoxic effects are prohibitive in the cultivation of transgenic fish as food. Therefore, a novel strategy to control reproduction in transgenic fish is urgently needed.

Primordial germ cells (PGCs) are the precursors of germ cells and appear early in an organism's development. PGCs migrate to the genital ridge where they differentiate into either sperm or eggs ${ }^{27}$. The dead end ( $d n d)$ gene is essential for the normal migration and survival of PGCs. When dnd is knocked down using dnd antisense morpholino oligonucleotide (MO), the migration of PGCs is disrupted and apoptosis of PGCs occurs. Zebrafish embryos devoid of $d n d$ develop into sterile male adults ${ }^{28-30}$. Therefore, one means of controlling fish reproduction is to inhibit gamete development by disrupting the migration of PGCs via the down-regulation of $d n d$ expression.

As a model organism, the zebrafish (Danio rerio) has been widely used in transgenic fish research ${ }^{26,31-35}$. The GAL4/UAS system, in which the yeast transcriptional activator GAL4 can activate transcription of effector transgenes under the control of GAL4-responsive upstream activator sequences (UAS), has been adapted for the precise control of gene expression patterns in vivo in zebrafish ${ }^{36}$. In this study, we develop a novel on-off strategy to control fish reproduction, which renders the offspring sterile but ensures that the parental generation remains fertile. We generated two transgenic lines using the GAL4/UAS inducible system: one line expressed the transcriptional activator GAL4, and the other line expressed the antisense $d$ nd RNA under the control of the $5 \times$ UAS effector. Both transgenic lines were fertile. However, in their hybrid offspring, GAL4 induced the $5 \times$ UAS-driven antisense $d n d$ transcription to knock-down the expression of endogenous dnd. This disrupted the migration of PGCs, brought about their apoptosis, and led to the sterility or reduced reproductive capacity of adults. Importantly, this novel method ensures the maintenance of transgenic lines that can serve as brood stocks alongside the production of sterile hybrid offspring that evade the problems of ecological risks that plague the use of transgenic fish.

\section{Results}

Endogenous $d n d$ expression was inhibited during early embryogenesis in the TG3 transgenic line. A schematic representation of the on-off strategy for controlling reproduction in transgenic fish using the GAL4/UAS inducible system and the dnd antisense knock-down technique is shown in Figure 1. The TG1 line expressed the transcriptional activator GAL4, driven by the CMV promoter (Fig. 1a). The TG2 line expressed antisense dnd RNA under the control of $5 \times$ UAS (Fig. 1b). The expression level of the UAS-regulated gene was related to the number of UAS repeat units ${ }^{36}$. We evaluated the effect of a range of UAS repeat units (from 1 to 11) on egfp transgene activation induced by GAL4, and found the expression level of $5 \times$ UAS: egfp to be strongest (Supplementary Fig. 1). Based on this result, the TG2 line was established with a transplant of $5 \times$ UAS: antisense $d n d$ to get the strongest expression of antisense $d n d$ in the hybrids of TG1 and TG2 lines (which are hereafter referred to as the TG3 line; Fig. 1c).

During the development of wild-type (WT) embryos, the expression level of endogenous $d n d$, nanos 1 and $t d r d 7$ mRNA was initially high then decreased rapidly (Fig. 2a-c). There were no significant differences in the endogenous dnd mRNA level between the TG3 and the WT lines at the stages of 1-cell, 1k-cell and oblong (Fig. 2a). Meanwhile, $d n d$ mRNA level in the TG3 line was markedly lower than that of the WT line at the stages of 50\%-epiboly and 3-somite (p $<0.01$; Fig. 2a). The $d n d$ gene encodes an RNA binding protein that can bind to the $3^{\prime}$ untranslated regions of nanos 1 and $t d r d 7$ mRNAs to protect them from miR-430-mediated repression ${ }^{37,38}$. Hence, the expression level of nanos 1 and $t d r d 7$ mRNA can mirror the level of the DND protein. Our study showed that the patterns of nanos 1 and $t d r d 7$ expression change were similar to those of $d n d$ during early development, both in the TG3 line and in the WT embryos (Fig. 2b, c). The nanos 1 and $t d r d 7$ mRNA levels were normal at the 1 -cell, $1 \mathrm{k}$ cell and the oblong stage in the TG3 line, but were significantly lower than those of the WT line at the $50 \%$-epiboly stage $(\mathrm{p}<0.05)$ and at the 3 -somite stage ( $\mathrm{p}<0.01$; Fig. $2 \mathrm{~b}, \mathrm{c}$ ). These results suggest that the endogenous dnd expression could be significantly repressed by GAL4/UAS induced antisense $d n d$ in the TG3 line during early embryogenesis.

PGC migration was impaired and the number of PGCs was reduced in TG3 embryos. The dnd gene is essential for the migration and survival of PGCs in zebrafish ${ }^{28}$. We labeled PGCs with antisense vasa probes to detect the migration and number of PGCs during the embryogenesis. At 4.3 hours post-fertilization (hpf), when compared with the normal distribution of PGCs seen in the majority of WT embryos $87 \%$ (141/166), about $72 \%(94 / 130)$ of TG3 embryos showed unusual distribution of some PGCs, with most being located far from the yolk syncytial layer (YSL; Fig. 2d). At $20 \mathrm{hpf}$ and $24 \mathrm{hpf}$, the number of PGCs which had migrated near the genital ridge varied among TG3 embryos. Moreover, when compared to the WT embryos the number of PGCs in TG3 embryos had visibly reduced in $79 \%(109 / 138)$ of the TG3 embryos at $20 \mathrm{hpf}$ and 67\% (127/155) of the TG3 embryos at $24 \mathrm{hpf}$. Furthermore, there were almost no PGCs remaining in about $14.8 \%$ (23/155) of the TG3 embryos at $24 \mathrm{hpf}$ (Fig. 2e).

In order to label the PGCs using green fluorescent protein (GFP) in living embryos, TG4-construction was undertaken by replacing the GFP element of TG1-construction using a red fluorescent protein (RFP) element (Fig. 3a). Then a TG5 line was derived by crossing the TG2 and TG4 lines. To label the PGCs, the 5' end-capped mRNA encoding GFP along with nanos1 3'UTR was then injected into the TG5 line and the WT embryos at the 1-cell stage. At $10 \mathrm{hpf}$ under a fluorescence stereoscope, the PGCs could be observed to converge into two clusters that were distributed symmetrically on both sides of the dorso axis in 83\% (332/400) of the WT embryos and 32\% (128/ 395 ) of the TG5 embryos, and were distributed far from the dorso axis in the remaining embryos. At $13 \mathrm{hpf}$, the PGCs lay close to the dorso axis and strongly arrayed in $86 \%(344 / 400)$ of the WT embryos and $36 \%(132 / 369)$ of the TG5 embryos, but lay far from the dorso 
a

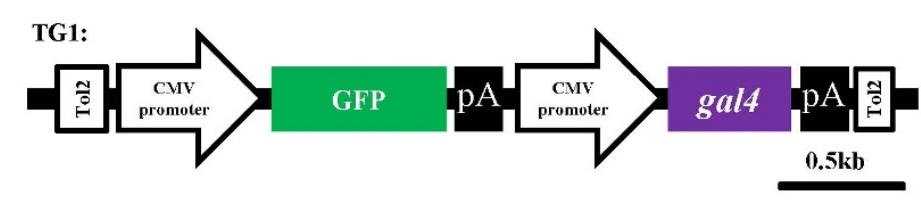

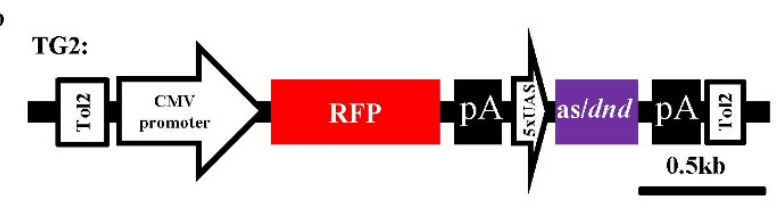

c

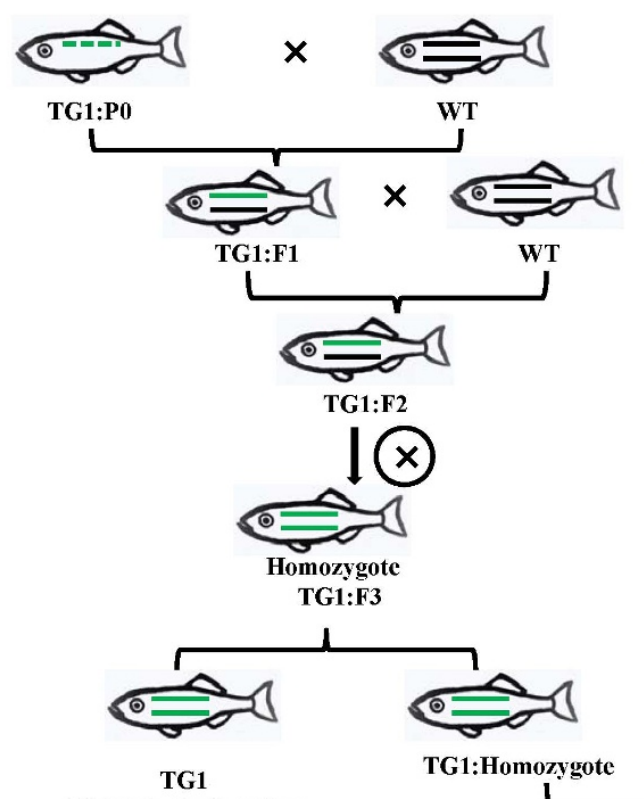

Maintain the breeders
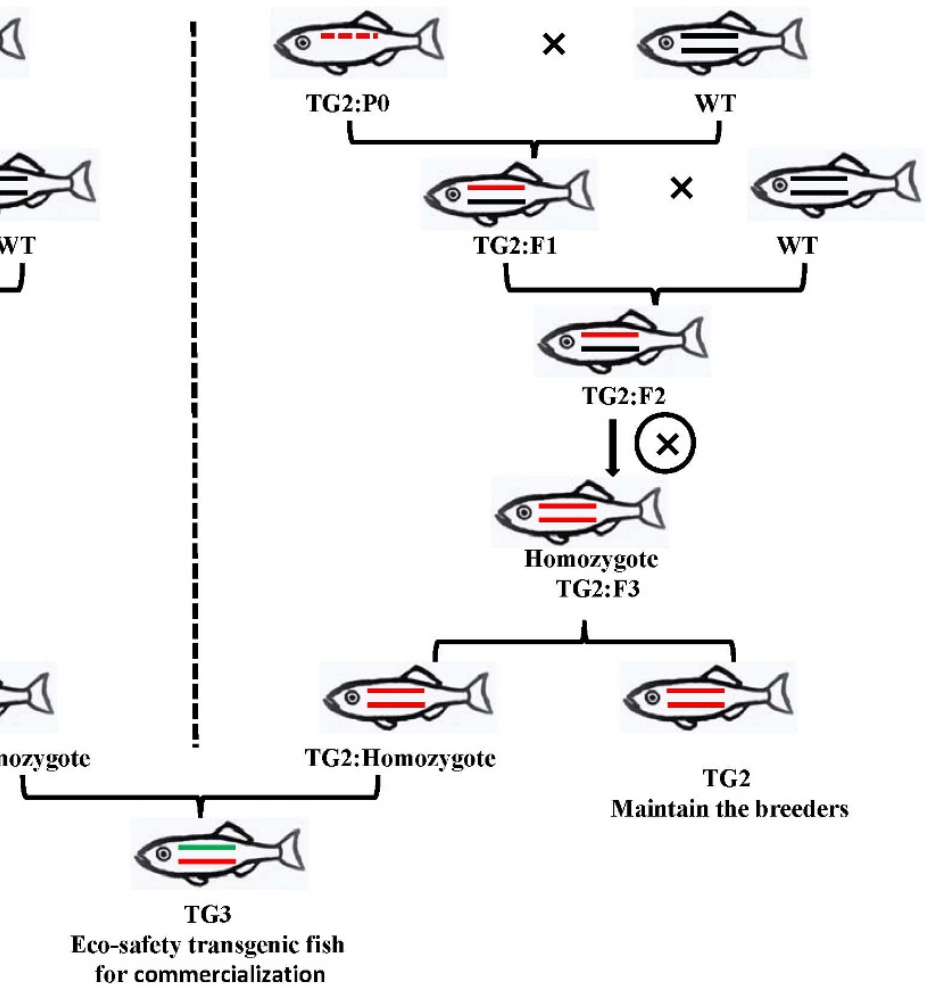

TG2:F1
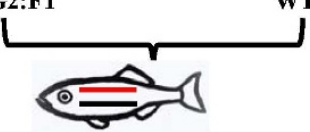

TG2:F2

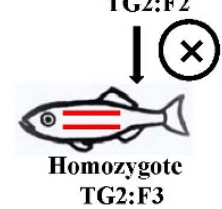

TG2:F3

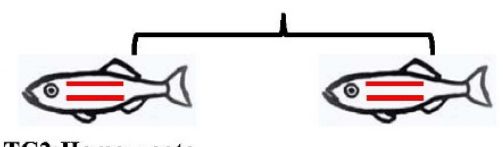

TG2

Maintain the breeders

for commercialization

Figure 1 Schematic representation of the on-off strategy to control transgenic fish reproduction. Schematic of expression gene cassette construct for generating (a) the TG1 line and (b) the TG2 line. (c) Flow chart showing how the parental generation maintains their fertility while their hybrid offspring become sterile. This figure was created using Adobe Photoshop CS3 Extended (Adobe Systems, USA) by Yunsheng Zhang.

axis in the remaining embryos (Fig. 3b). Meanwhile, at $20 \mathrm{hpf}$ and $24 \mathrm{hpf}$ there was a variable number of PGCs among the TG5 embryos, but a constant number of PGCs among the WT embryos. The number of PGCs visibly decreased in $80 \%$ (279/347) of the TG5 embryos at $20 \mathrm{hpf}$ and 62\% (169/273) of the TG5 embryos at $24 \mathrm{hpf}$ (Fig. 3b). No PGCs were detected in 17\% (47/273) of the TG5 embryos at $24 \mathrm{hpf}$ (Fig. 3c). We randomly selected 10 embryos with PGCs labeled by GFP from the TG5 and the WT lines at $24 \mathrm{hpf}$, and observed that the GFP fluorescence intensity of the PGCs within the WT embryos was four times that of the PGCs within the TG5 embryos (Fig. 3d).

To confirm whether the PGCs underwent apoptosis, 5'endcapped mRNA encoding GFP followed by nanos1 3'UTR was injected into the TG5 and the WT embryos at the 1-cell stage. The embryos were then analyzed for terminal deoxynucleotidyl transferase-mediated dUTP-biotinnick end labeling assay (TUNEL assay). The results showed that part of the PGCs underwent apoptosis in $13.4 \%(19 / 142)$ of the TG5 embryos at $20 \mathrm{hpf}$ and $16 \%(20 / 124)$ of the TG5 embryos at $24 \mathrm{hpf}$, while apoptosis of PGCs occurred in a further $2 \%(3 / 143$ and 3/126) of the WT embryos at $20 \mathrm{hpf}$ and 24 hpf (Fig. 3e).

TG3 adult fish were sterile or exhibited limited reproductive capacity. The majority of juvenile zebrafish reach sexually mature within three to four months ${ }^{39}$. In this study, we evaluated reproductive capacity at 4.5 months of age as at this age all the WT fish were able to be naturally inseminated. In this experiment, the fertilization rate of WT fish was $89.27 \%$. While all the TG3 males chased the WT females, $30.8 \%$ (53/172) failed to fertilize the eggs. About a third $(31 \% ; 15 / 48)$ of the TG3 females did not spawn after stimulus by the WT males. For convenience, fish that did not reproduce are termed TG3-1, while those that did reproduce are termed TG3-2. Although TG3-2 individuals could reproduce, the fertilization rate of TG3-2 males was significantly lower than that of the WT males (Table 1). Likewise, the relative fecundity of TG3-2 females was significantly lower than that of the WT females (Table 1).

There was no significant difference between the body weight of the TG3-1 and TG3-2 individuals, but the gonad somatic indices (GSI) of TG3-1 and TG3-2 were significantly lower compared to the WT individuals (Table 1). Gonadal histological slices were stained by hematoxylin-eosin (HE). The result showed that in the testes of WT fish the lobular cavities were filled with mature sperm that were aligned in a tight and orderly manner. In the testes of the TG3-1 males, lobular cavities were fewer in number and smaller than in the WT fish, and contained very little or no mature sperm. In TG3-2 individuals, only a portion of the lobular cavities lacked mature sperm.

In the ovaries of WT females, most oocytes were at stage III. However, in the ovaries of TG3-1 females, the majority of oocytes were at stage I or II, with only a few oocytes having developed to stage III. In ovaries of TG3-2 females, meanwhile, most oocytes were at stage III, but were loosely aligned and fewer in number (Fig. 4a). The results of the TUNEL analysis revealed that apoptosis occurred more frequently in the gonads of the TG3-1 and TG3-2 fish than in the WT fish, regardless of sex. The cells undergoing apoptosis were not evenly 


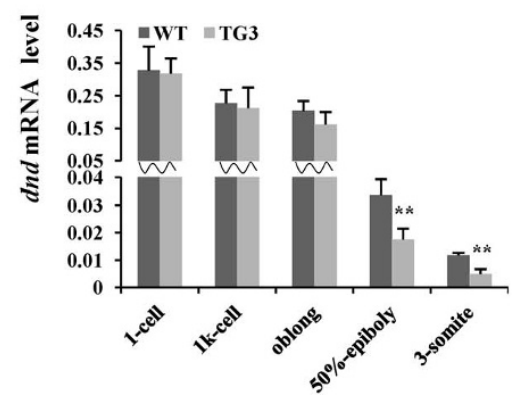

b

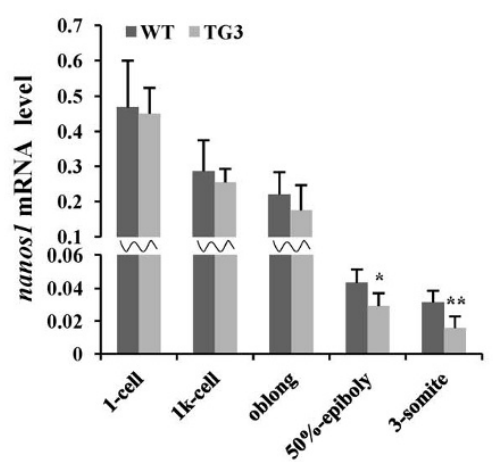

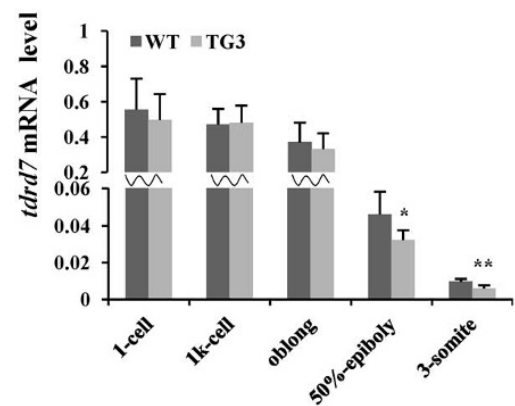

d

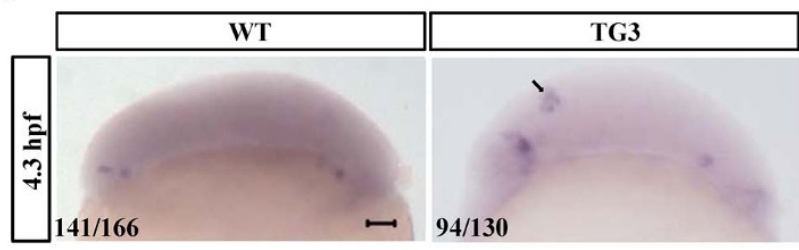
$141 / 166$

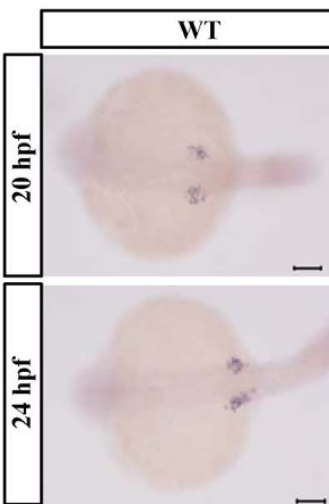

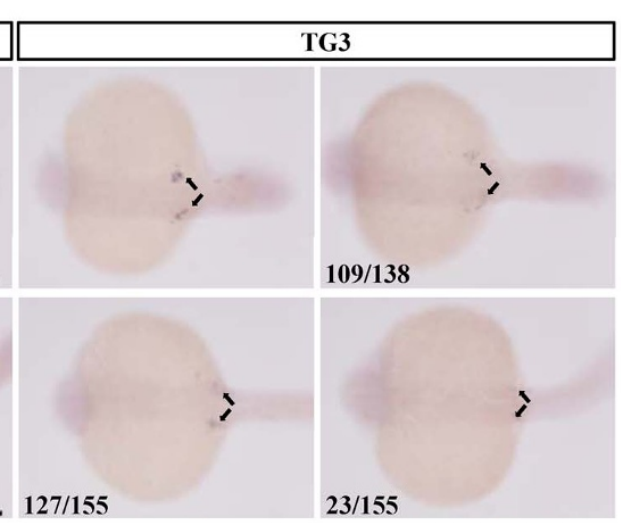

Figure $2 \mid$ The down-regulation of $\boldsymbol{d n d}$ results in the disrupted migration and reduced number of PGCs in the TG3 embryos. The mRNA level of (a) $d n d$, (b) nanos1, (c) $t d r d 7$ in the TG3 and the WT embryos at the 1-cell, 1k-cell, oblong, 50\%-epiboly, and 3-somite embryonic stages, validated by quantitative Q-PCR. The $2^{-\Delta C t}$ values were plotted as the relative mRNA level normalized to odc1. Values are represented as the mean \pm SEM. of five repeats. ${ }^{*} P<0.05, * * P<0.01$. (d) Vasa-positive PGCs were found close to the yolk syncytial layer (YSL) in the WT embryos. At 4.3 hours postfertilization (hpf) some PGCs (indicated by arrows) were far from the YSL in the TG3 embryos. (e) The number of vasa-positive PGCs decreased in the TG3 embryos compared with the WT embryos at $20 \mathrm{hpf}$ and $24 \mathrm{hpf}$ in the dorsal view. (Scale bars: $100 \mu \mathrm{m}$ ).

distributed, and the oocytes undergoing apoptosis were always at stage I or stage II (Fig. $4 \mathrm{~b}$ ).

The hybrid offspring exhibited methylation of the UAS and the mosaic expression of the UAS-regulated gene. The reproductive capacity of TG3 adults varied from complete sterility (TG3-1) to partial reproductive capacity (TG3-2). To understand the cause of this variation, we established the TG6 transgenic line, in which $5 \times$ UAS directly regulated the expression of egfp (Fig. 5a). Mosaic expression of egfp was observed in the embryos of the hybrid offspring of the TG4 and the TG6 lines, and varied among individuals (Fig. 5b). Mosaic expression of egfp was also observed in the testes and the ovaries of the hybrids, again with variation among individuals (Fig. $5 c, d$ ). We detected little dnd mRNA level in the gonads of TG3 adults (TG3-1 p $<0.01$; TG3-2 p $<0.05$ ), and significantly lower dnd mRNA level in the gonads of TG3-1 individuals than in TG3-2 individuals ( $p<0.05$; Fig. 5e, f). The gal4 mRNA level did not differ significantly between the TG3-1 and the TG3-2 adults (Fig. 5g, h). For the UAS each repeat (CGGAGTACTGTCCTCCGAG) contained two CpG sites, which were the targets of methylation. Hence, we examined the methylation status of $5 \times$ UAS and the minimal promoter E1b $(5 \times \mathrm{UAS}-\mathrm{E} 1 \mathrm{~b})$. The results showed that the $5 \times$ UAS-E1b sequence was susceptible to methylation in $77.5 \%$ of the TG3-1 males, $81.2 \%$ of the TG3-1 females, $88.3 \%$ of the TG3-2 male, and $90.8 \%$ of the TG3-2 females (Fig. 5i).

\section{Discussion}

The potential ecological risks posed by transgenic fish are a key impediment to their commercial use and development. Controlling the fertility of transgenic fish offers a solution to this problem. However, to date, a method of transferring the 'infertility' trait of the transgenic fish to its offspring while maintaining a self-propagating genetic line has not been established. The galactose regulated upstream promoter element (GAL4) is a transcriptional activator in yeast that contains not only the DNA-binding domain but also the transcriptional activation domain. The upstream active sequence (UAS) is a type of regulatory sequence like a eukaryotic enhancer that occurs in yeast. GAL4 can specifically recognize UAS and drive the transcription of genes following UAS: a system that has strong specificity and easily controlled ${ }^{36,40}$. In our study, TG1 and TG2 transgenic lines were generated using this GAL4/UAS system with $d n d$ as the target gene. We have shown that the expression of $d n d$ mRNA was significantly suppressed, resulting in disrupted migration of the PGCs and their apoptosis in the hybrid embryos. This process led either to sterility (TG3-1) or to poor reproductive ability (TG3-2) in adults. Our novel strategy (which ensures that the parental transgenic generation remains fertile while their hybrid offspring become sterile) can effectively maintain the valuable traits of transgenic fish while avoiding issues of ecological risk via the production of sterile offspring.

The fact that both sterile individuals (TG3-1) and individuals with poor reproductive ability (TG3-2) appeared in the TG3 line is likely associated with the methylation of UAS sequence. Genes of interest driven by $5 \times$ UAS in the Gal4/UAS system showed strong expression in stable transgenic zebrafish lines ${ }^{41,42}$. However, repetitive sequences attract methylation. Li and colleagues reported the methylation of $5 \times$ UAS in a human cell culture system ${ }^{43}$. Moreover, Engineer and colleagues found transcriptional silencing of genes 


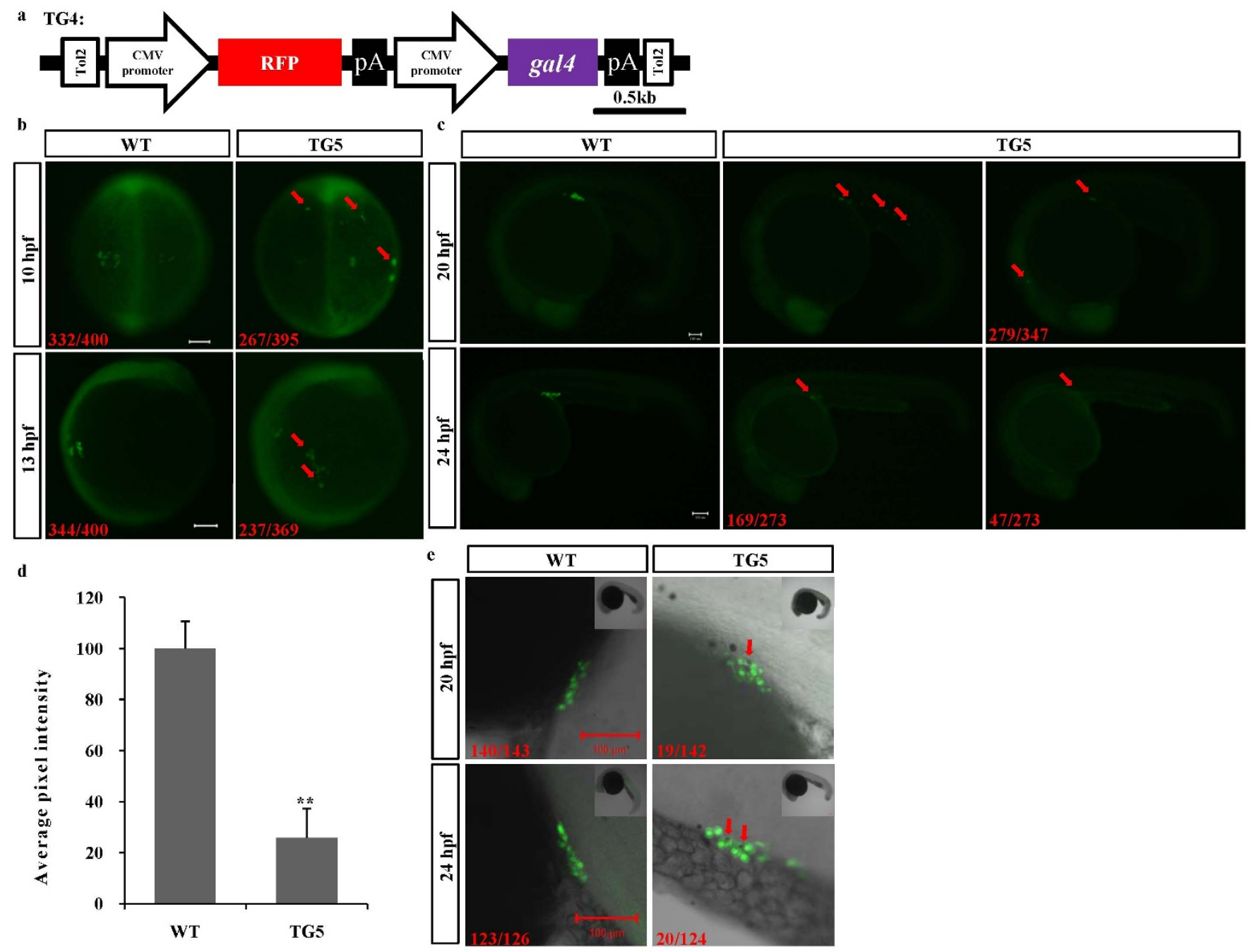

Figure 3 Validation of the migration, number and apoptosis of PGCs in the TG5 and the WT embryos. (a) Schematic of the expression gene cassette of the plasmids used to establish the TG4 line. (b) A part of PGCs labeled by GFP (red arrows) migrated abnormally or lagged behind in the TG5 embryos at $10 \mathrm{hpf}$ and $13 \mathrm{hpf}$, compared with the WT embryos. (c) The number of PGCs decreased in the TG5 embryos at 20 hpf and 24 hpf compared with the WT embryos. The red arrow indicates the location of PGCs. (d) WT embryos contained approximately four times the number of PGCs as the TG5 embryos (detected by GFP fluorescence intensity of PGCs at $24 \mathrm{hpf}(\mathrm{n}=10)$. (e) Apoptosis of some PGCs was detected (red arrows) within some of the TG5 embryos. (Scale bars: $100 \mu \mathrm{m})$.

driven by $5 \times$ UAS in transgenic Arabidopsis, and they suggested that the silencing is due to methylation, possibly at the level of $5 \times$ $\mathrm{UAS}^{44}$. In zebrafish, transcriptional repression was correlated with the $\mathrm{CpG}$ methylation of $14 \times \mathrm{UAS}^{45}$, and single insertions containing $4 \times$ UAS were found to be far less susceptible to methylation than insertions containing $14 \times \mathrm{UAS}^{46}$. In our study, we detected the methylation of $5 \times$ UAS in TG3 transgenic zebrafish according to a reported method ${ }^{47}$. Methylation of the $5 \times$ UAS-E1b sequence appeared in the gonads of the TG3 line, and there was significantly less methylation of the $5 \times$ UAS-E1b sequence in the TG3-1 indivi- duals than in the TG3-2 individuals. Accordingly, the level of endogenous $d n d$ mRNA was significantly lower in the gonads of the TG31 line compared with the TG3-2 line. Moreover, the gal4 gene driven by the CMV promoter was found to be effectively expressed in the gonads of TG3 transgenic fish and the level of gal4 mRNA did not differ between the TG3-1 and TG3-2 lines; further, GFP or RFP under the control of the CMV promoter was strongly expressed in the next generation (F5) of these transgenic lines (see Supplementary Fig. 2a). These findings indicate that the CMV promoter is active in transgenic zebrafish. The mosaic expression of the egfp gene regu-

Table 1 | Body weight, gonad somatic index (GSI), fertilization and relative fecundity ofWT, TG3-1, and TG3-2 fish. **p $<0.01$ vs. WT; \#\# $\mathrm{p}<0.01$ vs. TG3-1

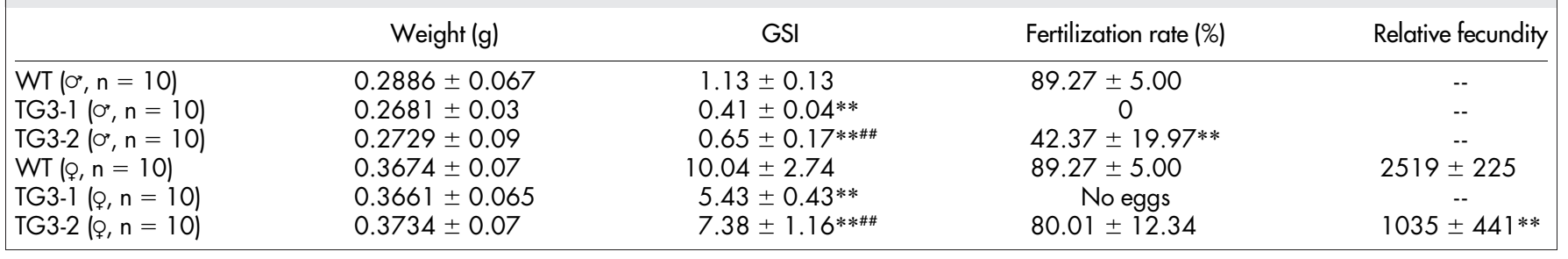


a

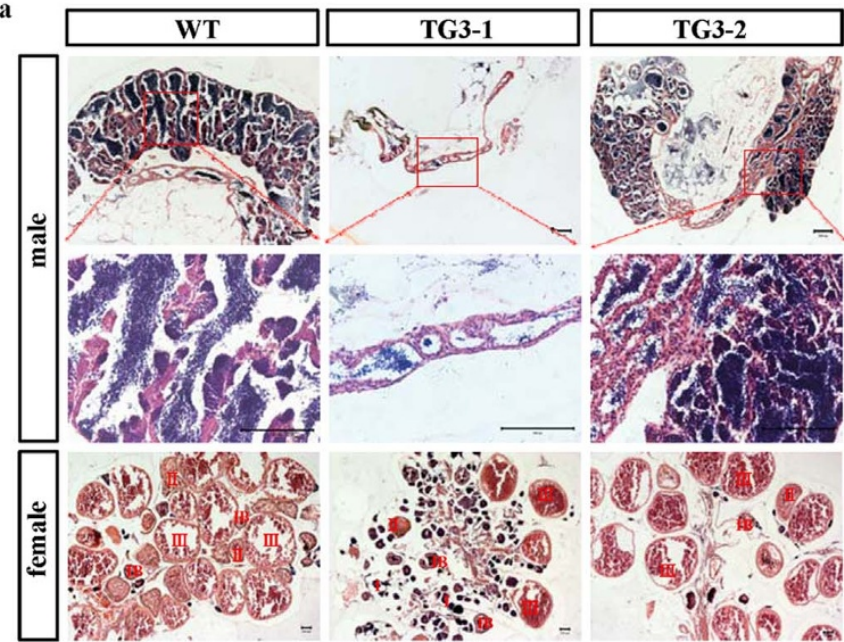

b

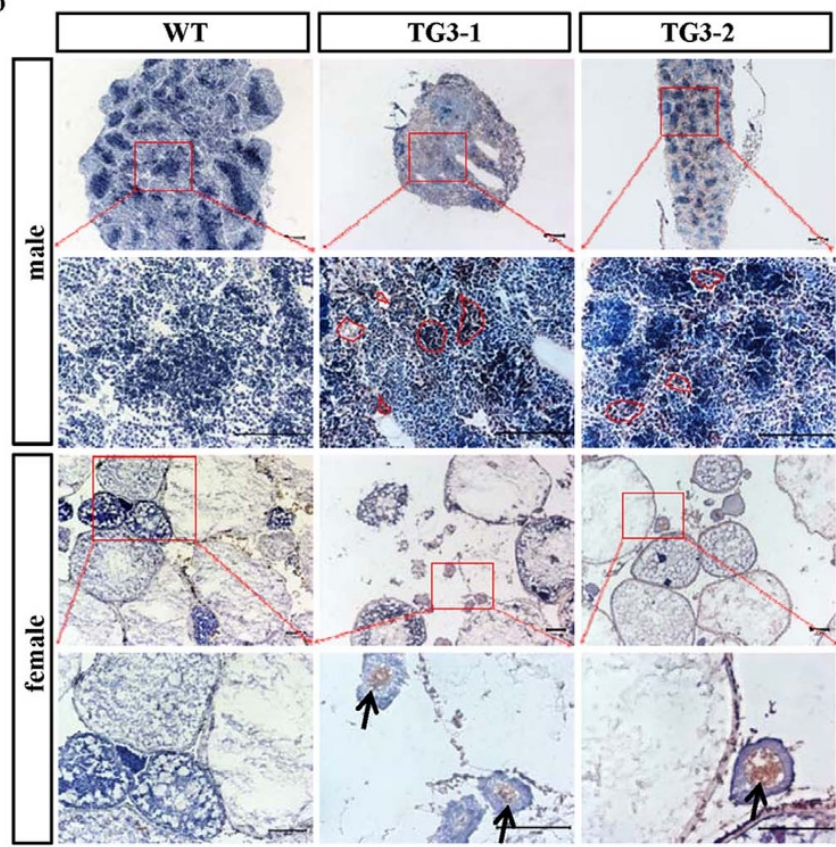

Figure 4 Histology and apoptosis analysis of the gonads of the TG3 adults. (a) Histology of the testes and ovaries from WT, TG3-1 and TG3-2 individuals by $\mathrm{HE}$ staining at 4.5 months of age. The lobular cavities in gonad of TG3-1 and TG3-2 males were observed to be in a single-row, were smaller than those of the WT males and contained none or very few mature sperm. When compare to the WT females, most oocytes were at stage I and widely dispersed throughout the gonads of the TG3-1 female fish, and at stage III and loosely arrayed in the gonads of the TG3-2 females. (b) Results of TUNEL staining of the testes and ovaries from the WT, TG3-1 and TG32 individuals. Multiple clusters of apoptotic spermatocytes were present in the testes of TG3-1 and TG3-2 males and some apoptotic oocytes were also present in the ovaries of TG3-1 and TG3-2 females. The red dotted line and black arrows indicate germ cells that were undergoing apoptosis. (Scale bars: $100 \mu \mathrm{m})$.

lated by the $5 \times$ UAS occurred not only in the larvae but also in the gonads of adults and was variable among individuals. The mosaic expression of UAS-regulated genes has also been reported by other researchers, and similarly attributed to the methylation of the $\mathrm{UAS}^{45,46}$. The mosaic expression of the UAS-regulated gene is even known to occur in stable transgenic lines ${ }^{48}$. Different tissues and different cells from the same tissue have different methylation levels ${ }^{45}$, and the methylation level of the promoter is also known to vary during an organism's development ${ }^{49}$. In mammals, DNA methylation levels differ among individuals and even in different cells $^{50,51}$. In humans, meanwhile, almost every sperm cell has its own unique pattern of methylation ${ }^{52}$. We surmised that the patterns of methylation in zebrafish might be similar to mammals. Germ cells from different individuals or from the same individual might exhibit different levels of UAS methylation leading to different levels of mosaic expression of antisense $d n d$ RNA. These differences could generate variation in the numbers of germ cells undergoing apoptosis in the gonads of the TG3 individuals, resulting in TG3 embryos that develop into adults with different reproductive capacities. To our knowledge, this is the first time methylation of $5 \times$ UAS has been identified in zebrafish, so these findings may prove useful for research on the regulation of gene expression with the Gal4/UAS system in zebrafish.

PGCs in zebrafish are located at the blastoderm layer and close to the yolk syncytial layer (YSL) and start to migrate at the dome embryonic stage. By the shield stage, PGCs have migrated close to the dorso axis at which point they begin to cluster. By the 8-somite stage, the PGCs have gathered into two clusters distributed symmetrically on both sides of the dorso axis ${ }^{53,54}$. In our study, some PGCs remained far from the YSL in the majority of the TG3 embryos at $4.3 \mathrm{hpf}$, which suggested that $d n d$ expression had been inhibited by the antisense dnd RNA. Continuous transcription of the antisense $d n d$ disrupted the migration of the majority of PGCs at $10 \mathrm{hpf}$ and 13 hpf. We then observed that the PGCs underwent apoptosis at $20 \mathrm{hpf}$ and $24 \mathrm{hpf}$, resulting in few or no PGCs migrating to the genital ridge. Similar results have been reported to occur when $d n d-\mathrm{MO}$ was injected into zebrafish embryos, resulting in a reduction or even disappearance of PGCs in the embryo stage and males that were sterile or exhibited very low reproductive capacity ${ }^{28-30}$. In general, fish fry containing no or few PGCs develop into males. For example, when the number of germ cells was reduced in ziwi mutants, the fish developed into sterile males ${ }^{55}$. In addition, transplantation of single PGC into embryos with no PGCs created male adults with a unilateral gonad ${ }^{56}$. However, in our study, it was interesting to note that the TG3 embryos developed into sterile and low reproductive adults. In zebrafish, dnd is a maternal gene and its expression can also be detected in the gonads of adult fish ${ }^{28}$ (see Fig. 2a, Fig. 5e, f). In MO-injected embryos, this inhibitory effect is strongest upon injection with $\mathrm{MO}$ and grows gradually weaker due to the degradation of $\mathrm{MO}^{57}$, dnd mRNA level decreases rapidly after fertilization (see Fig. 2a). Thus almost no PGCs migrate to the genital ridge and all the fry develop into males. However, in our study, transcription of the gene (regulated by UAS) became gradually stronger when induced by GAL4 (see Supplementary Fig. 2b), which could explain the lower levels of $d n d$ mRNA at the 50\%-epiboly and 3somite stages, but not at the 1-cell, 1k-cell and oblong stages. Additionally, the mosaic expression of the UAS-regulated gene appeared in our research, another factor that could result in the successful migration of some PGCs to the genital ridge. Indeed, the presence of some PGCs is essential for female differentiation in zebrafish $^{58}$, and explains why some females appeared in the TG3 line. Our results also suggest that $d n d$ might play an important role in the survival of germ cells in adult zebrafish.

The mechanism by which the number of germ cells affects sex differentiation remains unknown in zebrafish. Based on the histological features of their gonads, zebrafish are thought to be a bony fish that exhibits a protogynous hermaphrodite at the larval stage ${ }^{59}$. 'Juvenile ovaries' are formed in all fries at 10-14 days post-fertilization (dpf), and oocyte apoptosis, which marks the transformation of 'juvenile ovaries' into testes occurs in some individuals at $21 \mathrm{dpf}^{60}$. In ziwi mutant zebrafish, the number of germ cells has been reported to decrease rapidly or disappear before $21 \mathrm{dpf}$, such that all fry develop into sterile males ${ }^{55}$. In zebrafish, the mutation of fancl causes apoptosis of the oocytes that are undergoing meiosis and leads to femaleto-male sex reversal ${ }^{61}$. Wong et al. (2011) transplanted ovarian germ 

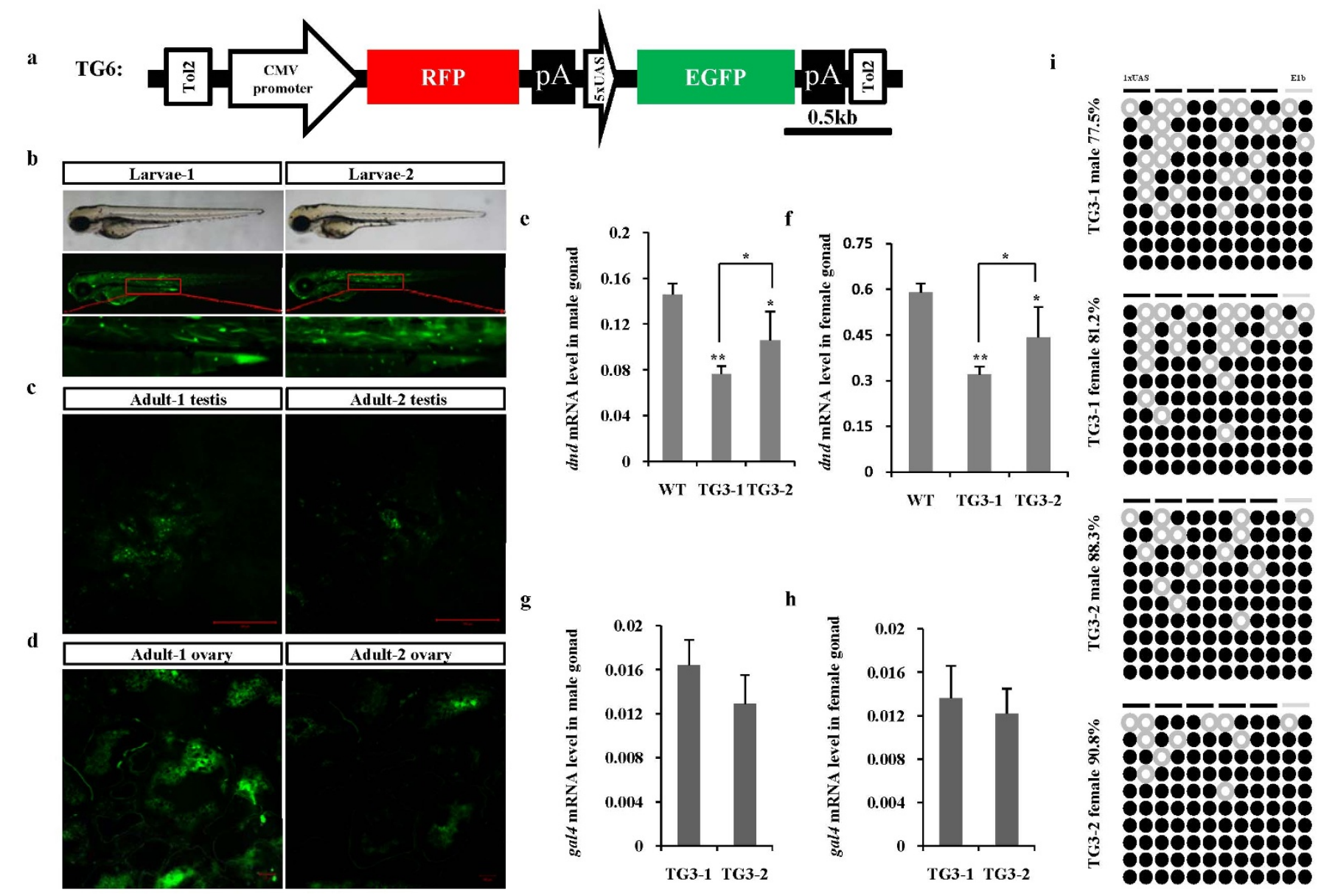

Figure 5 Mosaic expression of egfp regulated by $5 \times$ UAS and methylation analysis of $5 \times$ UAS-E1b by bisulfite sequencing. (a) Schematic of the expression gene cassette of the plasmids used to establish the TG6 line. (b) Variable mosaic expression of the $5 \times$ UAS-regulated egf $p$ gene was detected in the offspring of the TG4 and TG6 lines (at $60 \mathrm{hpf}$ ). The variable mosaic expression of the $5 \times$ UAS-regulated egfp gene was detected in (c) the testes and (d) the ovaries of the offspring of the TG4 and the TG6 lines (at 3 months of age). (Scale bars: $100 \mu \mathrm{m}$ ). The expression of $d n d$ in (e) the male gonads and (f) female gonads of the WT, TG3-1 and TG3-2 lines was validated by quantitative PCR (Q-PCR). The expression of gal4 in (g) the male gonads and (h) the female gonads of TG3-1 and TG3-2 individuals was validated by Q-PCR. The $2^{-\Delta C t}$ values were plotted as the relative mRNA level normalized to odc1. Values are represented as the mean \pm SEM of four repeats. ${ }^{*} P<0.05,{ }^{* *} P<0.01$. (i) DNA from the gonads of the TG3-1 and TG3-2 adults (at 4.5 months of age) was subjected to bisulfite sequencing. CpG methylation patterns in the $5 \times$ UAS-E1b are indicated on the horizontal axis. The first ten CpG sites resided within the $5 \times$ UAS, the last two CpGs sites resided within the E1b promoter. Ten clones were tested and the results are shown on the vertical axis. Black circles represent methylated sites.

cells $(\mathrm{N}=12 \pm 4.7)$ obtained from three-month-old adults into two-week-old sterile larva and reported that the larva developed into both males and females, of which a few males had low fertility levels while all the females were sterile ${ }^{62}$. In our study, some sterile and low reproductive female adults also appeared in the TG3 line. According to the above lines of evidence, we hypothesized that when the reduction of germ cells occurs before sex differentiation, more individuals differentiate into males. When the reduction of germ cells occurs after sex differentiation, then if the germ cells are absent female-to-male sex reversal transpires. However, the presence of just a few germ cells in the ovaries was evidently enough to maintain the female characteristics and not trigger female-to-male sex reversal. The reasons for this might be that the process of de-differentiation (and re-differentiation) in specialized cells of the gonad tissues (such as sertoli cells in testis and granulosa cells in ovarian) is difficult.

Our research has shown that antisense $d n d$ RNA regulated by $5 \times$ UAS can inhibit the expression of endogenous $d n d$. We observed that this process was affected by the methylation of UAS and resulted in partial sterility of the adults. However, we expect that the use of gene editing tools such as TALEN or CRISPR/Cas9 to refine the strategy implemented in this study (namely, to induce sterility in the offspring of two viable transgenic parental lines) will ensure that all hybrid offspring are sterile. This is because these tools enforce complete knockout of the $d n d$ gene, allowing for the establishment of the reproductive switch in fish.

The novel reproductive containment strategy we have developed in this study will be of great use in controlling the reproduction capacity of introduced fish. The introduction of exotic species is a traditional way of obtaining fish with valuable traits for aquaculture, but this process can cause extensive economic and environmental harm. For example, it is estimated that up to 5.4 billion dollars are lost annually in USA due to the negative impacts of exotic fish ${ }^{63}$. Another notable example is the extent of environmental damage caused by the introduction of the Asian carp in the USA. Recently, Thresher et al., (2014) reported a successful means of controlling invasive fish by reducing the effective population size of females ${ }^{64}$. The controllable on-off strategy for the reproductive containment of fish that we have developed provides a novel means of controlling the ecological and economic risks inherent to fish introductions. 


\section{Methods}

Fish. AB strain zebrafish were maintained and raised in recirculation systems at about $28.5^{\circ} \mathrm{C}$ with 14 hours light and 10 hours dark each day. All animal experiments were conducted in accordance with the Guiding Principles for the Care and Use of Laboratory Animals and were approved by the Institute of Hydrobiology, Chinese Academy of Sciences (Approval ID: keshuizhuan08529).

Generation of the transgenic zebrafish lines. The GAL4 and $5 \times$ UAS sequence were amplified by PCR from the TK5 $\times$ C vector ${ }^{36}$. The GAL4 sequence was cloned into lab stocks of the pSK-GFP (Tol2-CMV-GFP-pA-CMV-MCS-pA-Tol2) and the pSK-RFP (Tol2-CMV-RFP-pA-CMV-MCS-pA-Tol2) vectors to get TG1construction (Tol2-CMV-GFP-pA-CMV-gal4-pA-Tol2; Fig. 1a) and TG4construction (Tol2-CMV-RFP-pA-CMV-gal4-pA-Tol2; Fig. 3a). The $5 \times$ UAS sequence was cloned into the pSK-RFP vector for the construction of the pSK-RFP-5 $\times$ UAS (Tol2-CMV-RFP-pA-5 $\times$ UAS-MCS-pA-Tol2) vector. The primers used were GAL4-s (5'-CCCAAGCTTGCCGCCACCATGAAACTGCTC- $3^{\prime}$ ) and GAL4a (5' -CCCCCCGGGGGGAGGATGTCCAGGTCGTAGTC-3'); $5 \times$ UAS-s $\left(5^{\prime}\right.$ ACGCGTCGACGTCGCGGAGTACTGTCCTCCGAG- $3^{\prime}$ ) and $5 \times$ UAS-Elb-a (5'-CCCAAGCTTGGGAAAGTGAGGCTGAGACGCGATGCTCGGAGGACAGTACTCCG-3').

The antisense $d n d$ sequence (NCBI NM_212795.1, $-74 \mathrm{bp} \sim 257 \mathrm{bp}$ ) was amplified by PCR from the complementary DNA (cDNA) obtained from the 1-cell stage of the embryos of the WT fish. This was then cloned into the pSK-RFP- $5 \times$ UAS vector for TG2-construction (Tol2-CMV-RFP-pA-5 $\times$ UAS-as/dnd-pA-Tol2; Fig. 1b). The primers used were: Anti-dnd-s (5'-CCGCTCGAGCGGAATGACCTTTTCTTGACTTTTCC- $3^{\prime}$ ) and Anti-dnd-a (5'-CCGGAATTCCGGGAGGCGAAACTCGTAAATGG- $3^{\prime}$ ). The egfp gene (from pEGFP-C1) was cloned into the pSK-RFP- $5 \times$ UAS vector for TG6-construction (Tol2-CMV-RFP-pA- $5 \times$ UASEGFP-pA-Tol2; Fig. 5a). mRNA encoding Tol2 transposase (100 ng/ $\mu \mathrm{L})$ and Tol2 based constructs (TG1, TG2, TG4 and TG6; $50 \mathrm{ng} / \mu \mathrm{L}$ ) were co-injected into zebrafish embryos at the 1-cell stage. The positive embryos expressed fluorescence and could be screened with a fluoroscope (Olympus MVX10).

Quantitative PCR and in situ hybridization. Total RNA was extracted from the embryos (each sample contained 20-30 embryos) of the TG3 and the WT zebrafish at the 1-cell, $1 \mathrm{k}$-cell, oblong, 50\%-epiboly and 3-somite stages, using TRIzol reagent (Invitrogen). Total RNA was also extracted from the gonads of adult TG3 and WT zebrafish. After a 30 minute treatment with RNase-free DNase, the total RNA was reverse-transcribed to cDNA using Rever Tra Ace M-MLV (TOYOBO) with random primers. The cDNA samples were used for quantitative PCR (Q-PCR) analysis using $2 \times$ SYBR green real-time PCR mix (TOYOBO). The primers used for Q-PCR were: 5'-GTCAACAGACTCGGCTCTCC-3' /5'-GCACAAGGTTTGGATCACCT-3' for $d n d$, and $5^{\prime}$-TCCAAGGAAAAGCCGAAAT-3' $/ 5^{\prime}$-GGACGAATAAGCCAGTCAAAA-3' for gal4. The primers for Q-PCR analysis of nanos $1, t d r d 7$ and the reference ornithine decarboxxylase $1(o d c 1)$ gene were those described by Kedde et al., $2007^{37}$. PCR cycling conditions were as follows: $2 \mathrm{~min}$ at $95^{\circ} \mathrm{C}$, followed by 40 cycles of $15 \mathrm{~s}$ at $94^{\circ} \mathrm{C}, 15 \mathrm{~s}$ at $60^{\circ} \mathrm{C}$, and $40 \mathrm{~s}$ at $72^{\circ} \mathrm{C}$. The $2^{-\Delta \mathrm{Ct}}$ values were plotted as the relative mRNA level normalized to $o d c 1^{65}$. To label the PGCs, we used vasa (nucleotides 779-1284, GenBank accession number AB005147) as the marker gene ${ }^{66}$. In situ hybridization was performed as described in Thisse $\mathrm{Lab}^{67}$. Images were observed under a fluoroscope (Olympus MVX10) and captured using a digital camera (Nikon, MS-SMC) controlled by the ACT-2U software (Nikon).

Fluorescence microscopy and imaging of live PGCs. About 200 pg of RNA, consisting of a $5^{\prime}$ end-capped mRNA encoding GFP and a nanos $13^{\prime} \mathrm{UTR}$, was injected into the 1-cell stage embryos of the WT and the TG5 lines to visualize the PGCs. Images were observed under a fluoroscope (Olympus MVX10) and captured using a digital camera (Nikon, MS-SMC) controlled by ACT-2U software (Nikon). The average pixel intensity of the PGCs was measured using Image-pro plus6.0 software (Media Cybernetics, Inc.), and subtracted from the background signal. The injected embryos were collected and fixed in $4 \%$ paraformaldehyde (PFA) overnight at $4{ }^{\circ} \mathrm{C}$, then dehydrated in $30 \%$ sucrose solution for 48 hours. A TUNEL assay of the PGCs was performed using the In Situ Cell Death Detection Kit (Roche), according to the manufacturer's instructions. Images were captured under confocal microscope LSM 710 (Karl Zeiss).

Histological analysis and fertility assessment. Gonadal tissues were dissected from the TG3 and WT fish and fixed overnight in $4 \%$ PFA at $4{ }^{\circ} \mathrm{C}$ before being embedded in paraffin. The samples were sectioned at a width of $7 \mu \mathrm{m}$ and stained with hematoxylin-eosin (HE) using standard protocols. Developmental stages of oocytes were identified as described by Hu et al., $2010^{26}$. Fertility was assessed by pairing each TG3 male fish with a WT female fish (and vice versa) to mate naturally. If the WT females did not spawn or if their eggs were not fertilized after mating with the TG3 males the experiment was repeated 5 days later. TG3 males were considered sterile after three such failed attempts. Similarly, TG3 transgenic females were also considered sterile if they failed to spawn after three replicate mating experiments.

The fertilization rate and relative fecundity of TG3-2 adults were validated over a 15 day period. For each individual we calculated the gonad somatic index (GSI; gonad weight/body weight $\times 100$ ), the fertilization rate (fertilized eggs/total eggs $\times 100 \%$ ), and the relative fecundity [total eggs/body weight $(\mathrm{g})$ ].
TUNEL cell death assay of the gonadal tissues. Gonad tissues were dissected from 4.5 month-old adult TG3 fish and WT fish and embedded in Optimal Cutting Temperature (O.C.T. SAKURA). The samples were sectioned at a width of $7 \mu \mathrm{m}$ using a freezing microtome. The TUNEL cell death assay was performed using the In Situ Cell Death Detection Kit (Roche) according to the manufacturer's instructions. Images were observed under a fluoroscope (Olympus MVX10), and captured using a digital camera (Nikon, MS-SMC) controlled by ACT-2U software (Nikon) after hematoxylin re-dying.

DNA bisulfite sequencing. Gonad tissues were dissected from 4.5 month-old TG3 adults (TG3-1 males and females, TG3-2 males and females). Each sample contained six gonads of TG3 fish. The mixed genomic DNA was extracted using a genomic DNA kit (TIANGEN). The $5 \times$ UAS-Elb sequence was amplified using the primers $5^{\prime}$ TTTTTTTTATTGTATTTTAGTTGTGGT- ${ }^{\prime}$ and $5^{\prime}$-TAAAATTTTCATCAATCAAATCC- $3^{\prime}$. The PCR products were cloned into a pMD-18T vector (TAKARA) and ten clones from each sample were sequenced. The percentage of methylation of all sites $(\mathrm{CpG} \mathrm{n}=12)$ was counted in each sample.

Statistical analyses and image acquisition. T-tests were used to assess the significance of differences between the TG3 line and WT line in the following metrics: quantitative PCR data, body weight, GSI, fertilization rate and fecundity; $t$-tests were also used to assess the significance of differences between the TG5 line and WT line in fluorescence intensity and between TG3-1 and TG3-2 in quantitative PCR data, body weight, GSI and bisulfate. Images were taken and observation of the embryos and slices was performed using a fluoroscope (Olympus MVX10) and a confocal microscope LSM 710 (Karl Zeiss). The brightness and contrast of the images were processed using Adobe Photoshop (Adobe Systems, USA).

1. Holmlund, C. M. \& Hammer, M. Ecosystem services generated by fish populations. Ecol. Econ. 29, 253-268 (1999).

2. Neubauer, P., Jensen, O. P., Hutchings, J. A. \& Baum, J. K. Resilience and recovery of overexploited marine populations. Science 340, 347-349 (2013).

3. Worm, B. et al. Impacts of biodiversity loss on ocean ecosystem services. Science 314, 787-90 (2006).

4. Cressey, D. Future fish. Nature 458, 398-400 (2009).

5. Zhu, Z., Li, G., He, L. \& Chen, S. Novel gene transfer into the fertilized eggs of gold fish (Carassius auratus L. 1758). J. Appl. Lchthyol. 1, 31-34 (1985).

6. Maclean, N. \& Laight, R. J. Transgenic fish: An evaluation of benefits and risks. Fish. Fish. 1, 146-172 (2000).

7. Devlin, R. H., Biagi, C. A., Yesaki, T. Y., Smailus, D. E. \& Byatt, J. C. Growth of domesticated transgenic fish. Nature 409, 781-782 (2001).

8. Ledford, H. Transgenic salmon nears approva. Nature 497, 17-18 (2013).

9. Check, E. Environmental impact tops list of fears about transgenic animals Nature 418, 805-805 (2002).

10. Stokstad, E. Engineered Fish: Friend or Foe of the Environment? Science 297, 1797-1799 (2002).

11. Devlin, R. H., Sundstrom, L. F. \& Muir, W. M. Interface of biotechnology and ecology for environmental risk assessments of transgenic fish. Trends. Biotechnol. 24, 89-97 (2006).

12. Hu, W., Wang, Y. \& Zhu, Z. Progress in the evaluation of transgenic fish for possible ecological risk and its containment strategies. Sci. China. C. Life. Sci. 50, 573-579 (2007).

13. Hu, W. \& Zhu, Z. Integration mechanisms of transgenes and population fitness of GH transgenic fish. Sci. China. Life. Sci. 53, 401-8 (2010).

14. Marris, E. Transgenic fish go large. Nature 467, 259 (2010).

15. Van Eenennaam, A. L. \& Muir, W. M. Transgenic salmon: a final leap to the grocery shelf?. Natuer. 29, 706-710 (2011).

16. Wong, A. C. \& Van Eenennaam, A. L. Transgenic approaches for the reproductive containment of genetically engineered fish. Aquaculture 275, 1-12 (2008).

17. Fox, J. L. Transgenic salmon inches toward finish line. Nat. Biotechnol. 28, 1141-2 (2010).

18. Penman, D. J., Skibinski, D. O. F. \& Beardmore, J. A. Survival, growth rate and maturity in triploid tilapia. In Proc. World Symp. on Selection, Hybridization and Genetic Engeneering in Aquaculture 2, 277-288 (1987).

19. Ojolick, E. J., Cusack, R., Benfey, T. J. \& Kerr, S. R. Survival and growth of allfemale diploid and triploid rainbow trout (Oncorhynchus mykiss) reared at chronic high temperature. Aquaculture 131, 177-187 (1995).

20. Ozerov, M. Y. et al. High Gyrodactylus salaris infection rate in triploid Atlantic salmon Salmo salar. Dis. Aquat. Organ. 91, 129 (2010).

21. Fraser, T. W., Fjelldal, P. G., Hansen, T. \& Mayer, I. Welfare considerations of triploid fish. Rev. Fish. Sci. 20, 192-211 (2012).

22. Maclean, N. et al. Transgenic tilapia and the tilapia genome. Gene 295, 265-277 (2002).

23. $\mathrm{Hu}, \mathrm{W}$. et al. Antisense for gonadotropin-releasing hormone reduces gonadotropin synthesis and gonadal development in transgenic common carp (Cyprinus carpio). Aquaculture 271, 498-506 (2007).

24. Xu, J. et al. Defining Global Gene Expression Changes of the HypothalamicPituitary-Gonadal Axis in Female sGnRH-Antisense Transgenic Common Carp (Cyprinus corpio). Plos one 6, e21057 (2011).

25. Wong, T. T. \& Collodi, P. Inducible Sterilization of Zebrafish by Disruption of Primordial Germ Cell Migration. Plos one 8, e68455 (2013). 
26. Hu, S. Y. et al. Nitroreductase-mediated gonadal dysgenesis for infertility control of genetically modified zebrafish. Mar. Biotechnol. 12, 569-78 (2010).

27. Doitsidou, M. et al. Guidance of Primordial Germ Cell Migration by the Chemokine SDF-1. cell. 111, 647-659 (2002).

28. Weidinger, G. et al. dead end, a Novel Vertebrate Germ Plasm Component, Is Required for Zebrafish Primordial Germ Cell Migration and Survival. Curr. Biol. 13, 1429-1434 (2003).

29. Slanchev, K., Stebler, J., de la Cueva-Mendez, G. \& Raz, E. Development without germ cells: the role of the germ line in zebrafish sex differentiation. Proc. Nati. Acad. Sci. USA 102, 4074-9 (2005).

30. Liu, W. \& Collodi, P. Zebrafish dead end possesses ATPase activity that is required for primordial germ cell development. J. FASEB 24, 2641-50 (2010).

31. Li, Y. et al. Progranulin regulates zebrafish muscle growth and regeneration through maintaining the pool of myogenic progenitor cells. Sci. Rep. 3, 1176; DOI:10.1038/srep01176 (2013).

32. Guan, B. et al. Vitreoscilla hemoglobin (VHb) overexpression increases hypoxia tolerance in zebrafish (Danio rerio). Mar. Biotechnol. 13, 336-44 (2011).

33. Haas, P. \& Gilmour, D. Chemokine signaling mediates self-organizing tissue migration in the zebrafish lateral line. Dev. Cell. 10, 673-80 (2006).

34. Gong, Z. et al. Green fluorescent protein expression in germ-line transmitted transgenic zebrafish under a stratified epithelial promoter from keratin8. Dev. Dyn. 223, 204-15 (2002).

35. Hsu, C. C., Hou, M. F., Hong, J. R., Wu, J. L. \& Her, G. M. Inducible male infertility by targeted cell ablation in zebrafish testis. Mar. Biotechnol. 12, 466-78 (2010).

36. Distel, M., Wullimann, M. F. \& Koster, R. W. Optimized Gal4 genetics for permanent gene expression mapping in zebrafish. Proc. Nati. Acad. Sci. USA 106, 13365-13370 (2009).

37. Kedde, M. et al. RNA-binding protein Dnd1 inhibits microRNA access to target mRNA. Cell 131, 1273-86 (2007).

38. Mishima, Y. et al. Differential regulation of germline mRNAs in soma and germ cells by zebrafish miR-430. Curr. Biol. 16, 2135-2142 (2006).

39. Patton, E. E. \& Zon, L. I. The art and design of genetic screens: zebrafish. Nat. Rev. Genet. 2, 956-966 (2001).

40. Osterwalder, T., Yoon, K. S., White, B. H. \& Keshishian, H. A conditional tissuespecific transgene expression system using inducible GAL4. Proc. Natl. Acad. Sci. USA 98, 12596-601 (2001)

41. Asakawa, K. et al. Genetic dissection of neural circuits by Tol2 transposon mediated Gal4 gene and enhancer trapping in zebrafish. Proc. Nati. Acad. Sci. USA 105, 1255-1260 (2008).

42. Choe, S. K., Nakamura, M., Ladam, F., Etheridge, L. \& Sagerström, C. G. A Gal4/ UAS system for conditional transgene expression in rhombomere 4 of the zebrafish hindbrain. Dev. Dynam. 241, 1125-1132 (2012).

43. Li, F. et al. Chimeric DNA methyltransferases target DNA methylation to specific DNA sequences and repress expression of target genes. Nucleic. Acids. Res. 35 100-112 (2007).

44. Engineer, C. B., Fitzsimmons, K. C., Schmuke, J. J., Dotson, S. B. \& Kranz, R. G. Development and evaluation of a Gal4-mediated LUC/GFP/GUS enhancer trap system in Arabidopsis. BMC. Plant. Biol. 5, 9 (2005).

45. Goll, M. G., Anderson, R., Stainier, D. Y., Spradling, A. C. \& Halpern, M. E. Transcriptional silencing and reactivation in transgenic zebrafish. Genetics 182, 747-55 (2009)

46. Akitake, C. M., Macurak, M., Halpern, M. E. \& Goll, M. G. Transgenerational analysis of transcriptional silencing in zebrafish. Dev. Bio. 352, 191-201 (2011).

47. Deng, J. et al. Targeted bisulfite sequencing reveals changes in DNA methylation associated with nuclear reprogramming. Nat. Biotechnol. 27, 353-360 (2009).

48. Sagasti, A., Guido, M. R., Raible, D. W. \& Schier, A. F. Repulsive interactions shape the morphologies and functional arrangement of zebrafish peripheral sensory arbors. Curr. Biol. 15, 804-841 (2005).

49. Fang, X., Corrales, J., Thornton, C., Scheffler, B. E. \& Willett, K. L. Global and gene specific DNA methylation changes during zebrafish development. Comp. Biochem. Physiol. B Biochem. Mol. Biol. 166, 99-108 (2013).

50. Sandovici, I. et al. Interindividual variability and parent of origin DNA methylation differences at specific human Alu elements. Hum. Mol. Genet. 14 2135-43 (2005).

51. Lister, R. et al. Human DNA methylomes at base resolution show widespread epigenomic differences. Nature 462, 315-22 (2009).

52. Flanagan, J. M. et al. Intra- and interindividual epigenetic variation in human germ cells. Am. J. Hum. Genet. 79, 67-84 (2006).

53. Weidinger, G., Wolke, U., Koprunner, M., Klinger, M. \& Raz, E. Identification of tissues and patterning events required for distinct steps in early migration of zebrafish primordial germ cells. Development 126, 5295-5307 (1999).
54. Weidinger, G. et al. Regulation of zebrafish primordial germ cell migration by attraction towards an intermediate target. Development 129, 25-26 (2002).

55. Houwing, S. et al. A role for Piwi and piRNAs in germ cell maintenance and transposon silencing in Zebrafish. Cell 129, 69-82 (2007).

56. Saito, T., Goto-Kazeto, R., Arai, K. \& Yamaha, E. Xenogenesis in teleost fish through generation of germ-line chimeras by single primordial germ cell transplantation. Biol. Reprod. 78, 159-66 (2008).

57. Nasevicius, A. \& Ekker, S. C. Effective targeted gene 'knockdown' in zebrafish. Nat. Genet. 26, 216-220 (2000).

58. Siegfried, K. R. \& Nusslein-Volhard, C. Germ line control of female sex determination in zebrafish. Dev. Biol. 324, 277-87 (2008).

59. Maack, G. \& Segner, H. Morphological development of the gonads in zebrafish. J. FISH. BIOL. 62, 895-906 (2003).

60. Uchida, D., Yamashita, M., Kitano, T. \& Iguchi, T. Oocyte apoptosis during the transition from ovary like tissue to testes during sex differentiation of juvenile zebrafish. J. EXP. BIOL. 205, 711-718 (2002).

61. Rodriguez-Mari, A. et al. Sex reversal in zebrafish fancl mutants is caused by Tp53-mediated germ cell apoptosis. Plos. genet. 6, e1001034 (2010).

62. Wong, T. T., Saito, T., Crodian, J. \& Collodi, P. Zebrafish germline chimeras produced by transplantation of ovarian germ cells into sterile host larvae. Biol. Reprod. 84, 1190-7 (2011).

63. Pimentel, D., Zuniga, R. \& Morrison, D. Update on the environmental and economic costs associated with alien-invasive species in the United States. Ecol. Econ. 52, 273-288 (2005).

64. Thresher, R. et al. Sex-ratio-biasing constructs for the control of invasive lower vertebrates. Nat. Biotechnol. 32, 424-7 (2014).

65. Livak, K. J. \& Schmittgen, T. D. Analysis of Relative Gene Expression Data Using Real-Time Quantitative PCR and the $2^{-\Delta \Delta C T}$ Method. methods 25, 402-408 (2001).

66. Yoon, C., Kawakami, K. \& Hopkins, N. Zebrafish vasa homologue RNA is localized to the cleavage planes of 2- and 4-cell-stage embryos and is expressed in the primordial germ cells. Development 124, 3157-3165 (1997).

67. Thisse, C. \& Thisse, B. High-resolution in situ hybridization to whole-mount zebrafish embryos. Nat. protoc. 3, 59-69 (2007).

\section{Acknowledgments}

We greatly appreciate Mrs. Ming Li for raising the zebrafish. This work was supported financially by the " 863 " High Technology Project [grant number 2011AA100404], the National Natural Science Foundation [grant number 31325026] and the Key Research Program of the Chinese Academy of Sciences [grant numbers XDA08010106, KSCX2-EW-N-004, 2011FBZ19].

\section{Author contributions}

W.H., Z.Z., Y.Z. and J.D. conceived and designed research. Y.Z. and X.C. contributed to the construction of DNA constructs. H.X. conducted HE staining. Y.Z. and W.H. performed experiments, prepared figures $1-5$, modified methods and analyzed data. D.L. contributed to the sorting of images. Y.Z., W.H. and J.C. wrote the manuscript. All authors reviewed the manuscript.

\section{Additional information}

Supplementary information accompanies this paper at http://www.nature.com/ scientificreports

Competing financial interests: The authors declare no competing financial interests.

How to cite this article: Zhang, Y. et al. A controllable on-off strategy for the reproductive containment of fish. Sci. Rep. 5, 7614; DOI:10.1038/srep07614 (2015).

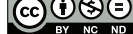

This work is licensed under a Creative Commons Attribution-NonCommercialNoDerivs 4.0 International License. The images or other third party material in this article are included in the article's Creative Commons license, unless indicated otherwise in the credit line; if the material is not included under the Creative Commons license, users will need to obtain permission from the license holder in order to reproduce the material. To view a copy of this license, visit http:/ creativecommons.org/licenses/by-nc-nd/4.0/ 